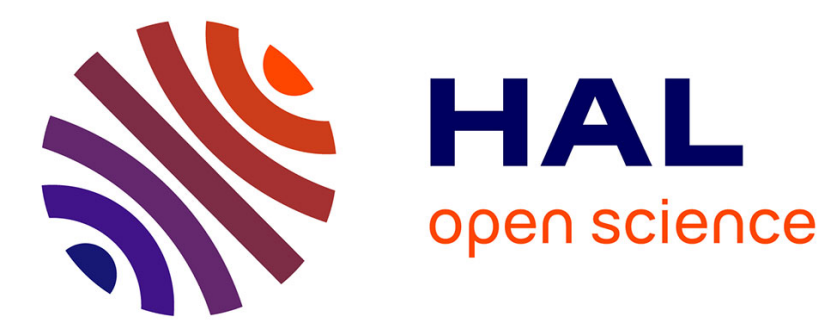

\title{
Dipole moment, hydrogen bonding and IR spectrum of confined water
}

François-Xavier Coudert, Rodolphe Vuilleumier, Anne Boutin

\section{To cite this version:}

François-Xavier Coudert, Rodolphe Vuilleumier, Anne Boutin. Dipole moment, hydrogen bonding and IR spectrum of confined water. ChemPhysChem, 2006, 7, pp.2464. 10.1002/cphc.200600561 . hal-00121925

\section{HAL Id: hal-00121925 \\ https://hal.science/hal-00121925}

Submitted on 22 Dec 2006

HAL is a multi-disciplinary open access archive for the deposit and dissemination of scientific research documents, whether they are published or not. The documents may come from teaching and research institutions in France or abroad, or from public or private research centers.
L'archive ouverte pluridisciplinaire HAL, est destinée au dépôt et à la diffusion de documents scientifiques de niveau recherche, publiés ou non, émanant des établissements d'enseignement et de recherche français ou étrangers, des laboratoires publics ou privés. 


\title{
Dipole moment, hydrogen bonding and IR spectrum of confined water**
}

\author{
François-Xavier Coudert,$^{\dagger}$ Rodolphe Vuilleumier ${ }^{\ddagger}$ and Anne Boutin ${ }^{\dagger *}$ \\ † Laboratoire de Chimie Physique, UMR 8000, Université Paris-Sud 11, F-91405 Orsay Cedex, France \\ ¥ Laboratoire de Physique Théorique de la Matière Condensée, UMR 7600, Tour 24, Boîte 121, \\ 4 place Jussieu, F-75252 Paris Cedex 05, France \\ * Corresponding author: anne.boutin@lcp.u-psud.fr
}

Keywords: confined water — hydrogen bonds — IR spectroscopy — CPMD — density functional calculations

Drastic changes can take place in the structure, dynamics, and thermodynamics of a fluid when it is confined to spaces of molecular dimensions, as compared to its bulk counterpart. Water confinement in the nanometer-scale channels and pores of inorganic open framework materials, such as zeolites, are of great scientific interest. Zeolites are crystalline aluminosilicates, with various and controlled pore sizes and connectivities. [1] From a practical point of view, water plays a key role in many applications, including ion-exchange and separation. From a more general point of view the interaction of water with solid surfaces is of key importance for many chemical and physical processes. However, our present understanding of interfacial or confined water at the molecular level is still very limited. Most of the experimental techniques are difficult to carry out in nanometer environments. Theoretical investigation can, thus, be of great help for a better understanding of confined water properties.

Classical simulations have recently been performed aimed at understanding the structure, dynamics,

\footnotetext{
${ }^{* *}$ We acknowledge the Institut du Développement et des Ressources en Informatique Scientifique (IDRIS) for computer time allocation and the french Agence Nationale pour la Recherche (ANR) for financial support. Many thanks are due to Alain Fuchs for his kind support and fruitful discussions.
} 
and thermodynamics of water confined in carbon micropores or nanotubes [2,3] and zeolites. [4-8] However, the quantum nature of bonding in water can only be captured by ab initio calculations. Elaborate treatments of the electronic density are usually limited to small clusters (or a few adsorbed molecules) and restricted to equilibrium structures. The Car-Parrinello Molecular Dynamics method (CPMD) [9] offers an alternative route to capture the electronic properties of molecules, as well as dynamics effects. Such DFT-based ab initio molecular dynamics calculations have been already applied to bulk water [10], interfacial water on Si surfaces [11], and zeolitic materials. [12,13] Most of the CPMD studies of confined water deal with the dynamics of one-dimensionnal water chains and helices in hydrophilic zeolites with narrow, non-connected, cylinder pores. $[12,13]$ We have used here this method for the first time to investigate water confined in a hydrophobic zeolite with large interconnected pores. The LTA zeolite displays supercages of diameter $\simeq 13 \AA$, connected to one another in a cubic symmetry by 8-ring windows of diameter $\sim 7 \AA$, and smaller sodalite cages of diameter $\simeq 7 \AA$. This zeolite is, thus, a good candidate for studying the effect of confinement in open structure hydrophobic nanoporous materials. We have studied the thermodynamic and electronic properties, and the vibrational infrared spectrum of the confined water. It is worth noticing that while infrared measurements are a powerful tool to explore the structure of confined fluids, IR spectra are often complicated to interpret. Vibrational spectra of confined fluids have been computed some years ago using normal mode analysis on minimal-energy structures or using polarizable forcefields and electrooptical models in classical molecular dynamics simulations. [14,15] Most studies use the velocity auto-correlation function that provides vibrational frequencies but not IR intensities, resulting in a quite different vision of the overall spectrum. This is the first time, to our knowledge, that the dipole moments and IR spectrum of confined water are calculated from ab initio dynamics. This technique was developed recently, based on an estimator of the dipole moment in periodic boundary conditions [16,17], and was shown to successfully reproduce infrared spectra of aqueous systems. [18-21]

We have first computed the water adsorption isotherm at $300 \mathrm{~K}$ following the Grand Canonical Monte Carlo methodology of Desbiens et al. [8,22] As it was experimentally observed on other siliceous zeolites [23], the all-silica LTA is found to be hydrophobic, with liquid intrusion occuring at $\sim 70 \mathrm{MPa}$. The maximum loading is found at $\sim 20$ water molecules per unit cell. Starting from these classical GCMC configurations we have performed CPMD simulations using plane-wave basis set and norm-conserving pseudopotentials on siliceous LTA with various hydration rates.

The effect of confinement on the molecular dipole of water is shown in Figure 1 for two molecular loadings: 15 and $20 \mathrm{H}_{2} \mathrm{O}$ molecules per unit cell of zeolite (one supercage plus one sodalite cage). For comparison, the water dipole of the gas phase water molecule and the distribution of dipole moments for 32 bulk water molecules computed using the same DFT and Car-Parrinello parameters are also plotted. The present bulk data are consistent with the original results of Silvestrelli et al. [24] The dipole distribution for 20 confined water molecules has a maximum at $2.9 \mathrm{D}$, close to the bulk water maximum found at $3.1 \mathrm{D}$, and displays a smaller band around $2 \mathrm{D}$, close to the gas phase value of $1.8 \mathrm{D}$. This latter peak corresponds 
to the water molecule located in the small sodalite cage. This seems to show that the polarization induced by the zeolite framework is quite small compared to the self-polarization of water fluid. From this point of view, this result is in good agreement with studies of smaller water clusters in siliceous silicalite by means of classical simulations using a polarizable water model. [5] However, unlike what was observed in silicalite, where the dipole distribution displays several peaks, the distribution observed in siliceous LTA is unimodal (apart from the water molecule in the sodalite cage). The multimodal dipole moment distribution was observed in silicalite but not in hydrophilic Na-LSX zeolite [25], and this has been interpreted in term of a intrinsic property of hydrophobic zeolites. Our results seem to indicate that a multimodal behaviour is related to the existence of multiple, strongly non-equivalent, adsorption sites in narrow pores (channels and intersections of silicalite, or the sodalite cage in the present case). In a more open structure, such as LTA supercage and LSX zeolite, the dipole moment distribution appears smoother.

The dependence of the water dipole moments on the number of adsorbed water molecules can also be compared from one zeolite structure to another. We observe an increase of about $0.04 \mathrm{D}$ per extra adsorbed molecule. This value is 20 times greater than the one previously observed in hydrophilic Na-LSX zeolite. [25] In this cationic aluminosilicate material, the electrostatic field induced by the zeolite is much stronger than in pure siliceous LTA zeolite, and an extra water molecule in Na-LSX has less effect on the overall field felt by other adsorbed water molecules. This is consistent with the fact that the apparent water dipole moment is larger than the bulk value in an aluminosilicate zeolite (because of the large electric field created by the framework) and smaller than the bulk value in an hydrophobic all-silica zeolite.

The water dipole moment is found to be smaller than in bulk water but larger than its gas phase value. In the large pores of LTA, water molecules self-organize through hydrogen bonds which build up a relatively large dipole. We have identified these hydrogen bonds by means of a geometric criterion involving the Acceptor, Donor, and Hydrogen atoms. [26] The number of hydrogen bonds among the 20 water molecules confined in the zeolite, calculated using this geometric criterion, is reported in Table 1. Very few waterzeolite hydrogen bonds are observed, while the difference in water-water $\mathrm{H}$ bonds ratio between bulk and confined water $(\sim 0.3)$ is small even though most of the water molecules are very close to the zeolite surface (less than $5 \AA$ ). In confined spherical hydrophobic pores, the 15-20 water droplet self-organizes and increases its cohesion energy. This is in keeping with the observation of cluster formation of $1 \mathrm{~nm}$ size in hydrophobic graphitic nanopores. [2]

The lifetime of hydrogen bonds defined from a purely geometric criterion are extracted from Figure 2. The water-water hydrogen bonds lifetime is found to be $1.5 \mathrm{ps}$, similar to the lifetime of such bonds in bulk water using the same methodology. Hydrogen bonds between confined water and the framework oxygen atoms, however, are found to have a lifetime of only 120 fs. Such OHO alignments are indeed observed but they don't correspond to true hydrogen bonds as proven by the absence of any water $\mathrm{O}-\mathrm{H}$ strain. In this specific case, usual geometric criteria are not appropriate to characterize hydrogen bonds. The nonexistence (or weakness) of zeolite-water hydrogen bonds between water and $\mathrm{SiO}_{2}$ framework is in keeping 
with experimental diffuse reflectance infrared Fourier transform spectroscopic (DRIFT) measurements in different zeolites which showed that zeolites with low Si:Al ratio exhibit only weak interaction between the water molecules and the zeolite oxygen atoms. [27]

Finally, we have studied the confinement effect on the water-zeolite infrared spectrum. We report in Figure 3 the infrared spectrum of the hydrated zeolite, as well as the separate IR spectra of the confined water and the zeolite. The zeolite spectrum is very similar to that of the dry solid, which we also computed (not shown here). Both spectra are in reasonnable agreement with the experimental data [28]. We do not observe any significant effect of the confined water on the vibrationnal spectrum of the zeolite framework and the two sub-systems are not correlated. Indeed, the total spectrum for the system matches the sum of the two separate spectra for water and the zeolite at frequencies higher than $300 \mathrm{~cm}^{-1}$.

Figure 4 shows the $\mathrm{OH}$-stretch region of both the confined and bulk water IR spectra. For confined water, the $\nu_{\mathrm{OH}}$ band appears to be composed of a large band and a narrower high-frequency peak. The existence of a narrow high-frequency peak in the $\mathrm{OH}$ stretch region of interfacial water has been revealed by the sum-frequency generation (SFG) technique, which is a highly surface-specific vibrational spectroscopy of liquid interfaces. This peak was attributed to non hydrogen-bonded $\mathrm{OH}$ bonds. [29] The dangling $\mathrm{OH}$ peak is more prominent for water near hydrophobic surfaces than in the hydrophilic case and, thus, can be used as a signature of hydrophobicity. The analysis of the velocity autocorrelation function of $\mathrm{OH}$ distances for $\mathrm{H}$ atoms involved in water-water hydrogen bonds and $\mathrm{H}$ atoms directed toward the zeolite (i.e. not involved in hydrogen bonds) demonstrates that the high frequency modes correspond to $\mathrm{OH}$ bonds that are not involved in the hydrogen bond network. Our results in the case of a more realistic system confirm the attribution of the high frequency $\nu_{\mathrm{OH}}$ peak to dangling hydrogens.

We have reported here DFT-based ab initio Molecular Dynamics results of water confined in a large pore LTA hydrophobic zeolite. We have studied the molecular dipole of confined water molecules, hydrogen bonding properties, and we have computed the system infrared spectrum.

It was found that the infrared spectrum of the structural zeolite is decorrelated from the confined water zeolite indicating very small interaction between the host and the guest species. This was confirmed by the study of hydrogen bonds which showed the absence of genuine water-zeolite hydrogen bonds. The water $\mathrm{OH}$-stretch band shows similar shape and frequency with respect to bulk water, except for a narrow peak at $2300 \mathrm{~cm}^{-1}$ attributed to $\mathrm{OH}$ bonds directed towards the zeolite. The lifetime of water-water hydrogen bonds in confined water was found to be similar to those in bulk water, indicating a similar H-bond strength within confined water.

Finally, we observed a slight decrease of the water dipole moment with respect to its bulk value. The resulting dipole moment is, however, still largely enhanced with respect to its gas phase value, due to hydrogen bonding. Further studies on other systems are now needed to understand the role of pure 
confinement with respect to possible specific interaction with the host structure and the role of confinement geometry. DFT-based ab initio MD also offers a possible route for studying the chemical reactivity of confined water.

\section{Computational Procedure}

The Molecular Dynamics simulations of anhydrous and hydrated zeolite were performed within the CarParrinello method [9] as implemented in the CPMD software package [30], using the Kohn-Sham formulation of DFT. We employed the gradient-corrected BLYP functional. [31,32] A planewave basis set with an energy cutoff of 70 Ry was used and only the valence electrons were explicitly considered, with semilocal norm-conserving Trouillier-Martins pseudopotentials.

The siliceous Linde Type A zeolite structure was taken from Ref [33]. The unit cell structure $\left(\mathrm{Si}_{24} \mathrm{O}_{48}\right)$ is cubic ( $\mathrm{Pm} \overline{3} \mathrm{~m}$ symmetry and $a=11.9 \AA)$ and contains one supercage and one sodalite cage. All hydrogen atoms were deuterated. A timestep of 7 a.u. $(0.17 \mathrm{fs})$ and a fictitious electron mass of 1000 a.u. were used. (A 1.5 ps test run with an electronic mass of 700 a.u. and a timestep of 4 a.u. showed no significant difference.) A first equilibration run of 1.2 ps was performed on each system, using a NoséHoover thermostat [34-36] with a frequency of $3800 \mathrm{~cm}^{-1}$. Production runs of $3 \mathrm{ps}$ in the microcanonical NVE ensemble were then performed. It is worth mentionning that the simulation time is rather short for a full phase-space sampling. This could lead to a large uncertainty of the hydrogen bonds lifetimes.

Trajectories of the total dipole moments were obtained using the Berry phase approach [16] and their autocorrelation function was used to compute the IR absorption coefficient, $\alpha(\omega)$, in the linear response theory. [18] To compute the dipole moments of individual water molecules, the electronic wavefunctions are replaced by the maximally localized Wannier functions [17] and the Wannier centers are assigned to each water molecule. $[19,21,37]$ A harmonic quantum-classical correction was then applied to the classical correlation function to account for the quantum aspects of the hydrogen motion, as was shown to be adequate in previous works $[20,21,38]$. The resulting infrared spectrum was then smoothed by a linear filter as described in Ref. [21]. 


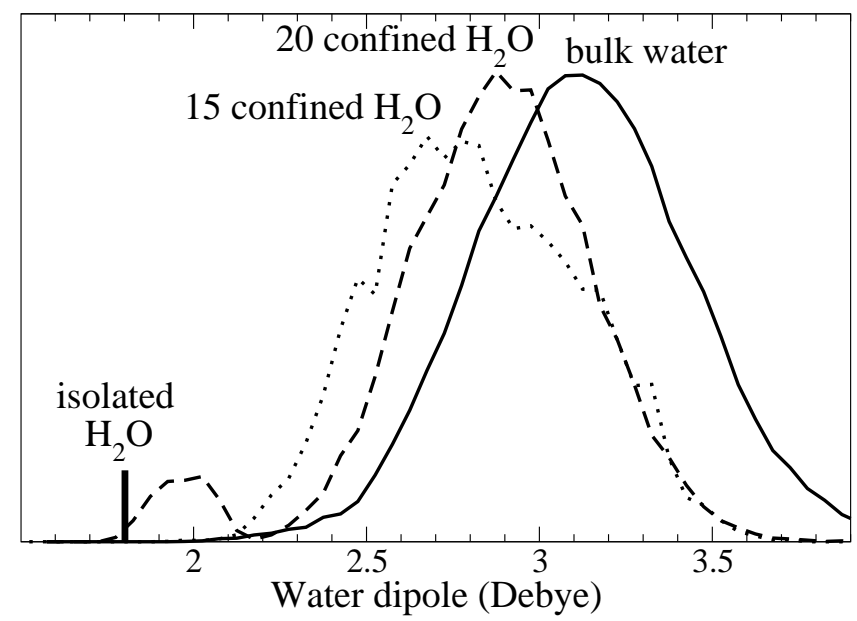

Figure 1: Distribution of the dipole moment for 20 (dashed line) and 15 water molecules (dotted line) confined in siliceous LTA zeolite, as compared to bulk water (solid line) and gas phase water $(\mu \simeq 1.8 \mathrm{D})$.

\begin{tabular}{ccc}
\hline & number & ratio \\
\hline water-water $\mathrm{H}$ bonds for 20 confined $\mathrm{H}_{2} \mathrm{O}$ & 30.8 & 1.54 \\
water-zeo $\mathrm{H}$ bonds for 20 confined $\mathrm{H}_{2} \mathrm{O}$ & 4.1 & 0.21 \\
water-water $\mathrm{H}$ bonds for $32 \mathrm{H}_{2} \mathrm{O}$ in bulk & 59.7 & 1.86 \\
\hline
\end{tabular}

Table 1: Number of water-water and water-zeolite hydrogen bonds in the zeolite supercage, as defined by the geometric criterion, and ratio over the total number of water molecules in the supercage. The number of hydrogen bonds in a 32 bulk water molecules system is also indicated for comparison.

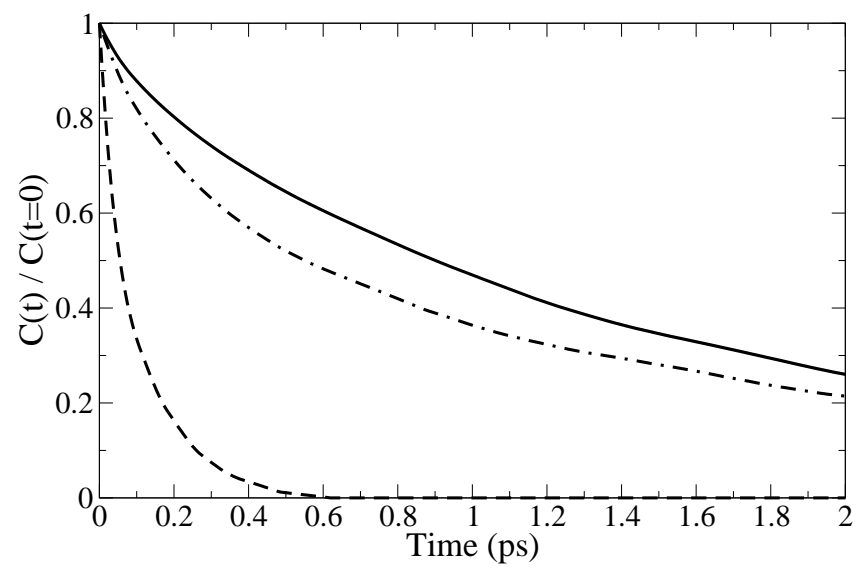

Figure 2: Autocorrelation function of hydrogen bonds defined from a purely geometric criterion: waterzeolite hydrogen bonds (dashed line), water-water $\mathrm{H}$ bonds for confined water (dash-dotted line) and bulk water (solid line). 


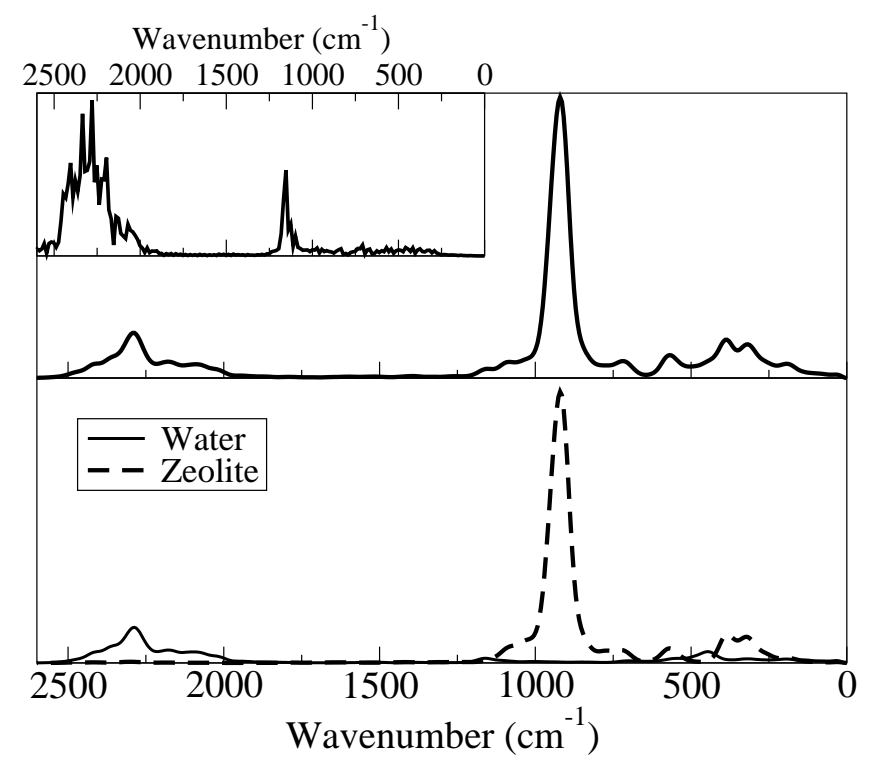

Figure 3: Infrared spectrum of the hydrated zeolite containing 20 deuterated water molecules: total IR spectrum (upper panel) and the separate spectra for the confined water (lower panel, solid line) and the zeolite (lower panel, dashed line). The power spectrum of the velocity autocorrelation function of the hydrated zeolite is shown in insert (upper left corner), for comparison purpose.

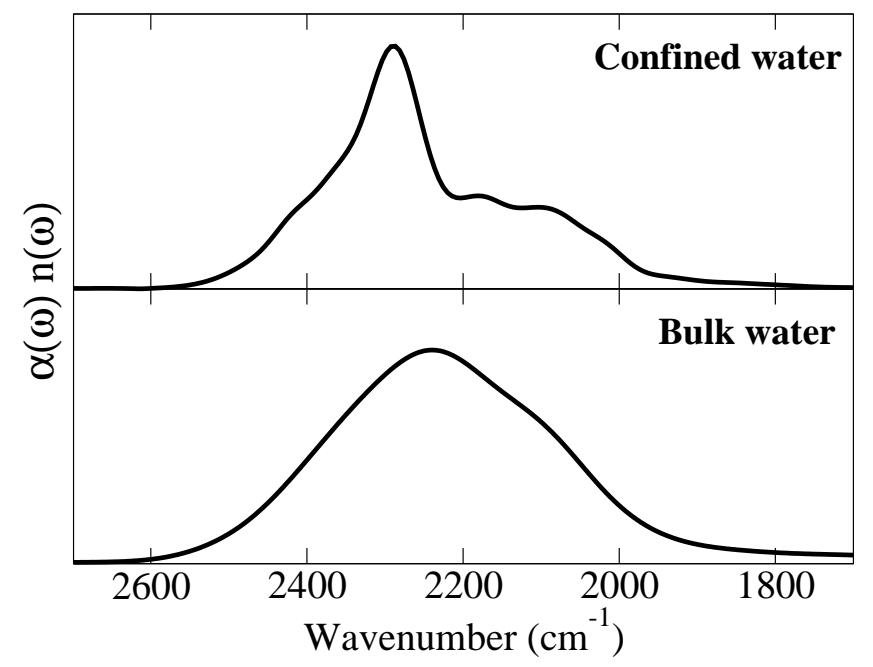

Figure 4: The $\mathrm{OH}$ stretch band of the infrared spectrum of the hydrated zeolite containing 20 deuterated water molecules (upper panel) compared to 32 bulk water molecules (lower panel). 


\section{References}

[1] S. M. Auerbach, K. A. Carrado, P. K. Dutta, , Eds. Handbook of Zeolite Science and Technology; Marcel Dekker, Inc. : New York, 2003.

[2] T. Ohba, H. Kanoh, K. Kaneko, J. Am. Chem. Soc. 2004, 126, 1560-1562.

[3] E. Mamontov, C. J. Burnham, S.-H. Chen, A. P. Moravsky, C.-K. Loong, N. R. de Souza, A. I. Kolesnikov, J. Chem. Phys. 2006, 124, 194703.

[4] P. Demontis, G. Stara, G. B. Suffritti, Studies in surface science and catalysis 2002, 142, 1931-1938.

[5] K. S. Smirnov, D. Bougeard, Chem. Phys. 2003, 292, 53-70.

[6] K. Shirono, A. Endo, H. Daiguji, J. Phys. Chem. B 2005, 109, 3446-3453.

[7] C. Beauvais, A. Boutin, A. H. Fuchs, C. R. Chimie 2005, 8, 485.

[8] N. Desbiens, A. Boutin, I. Demachy, J. Phys. Chem. B 2005, 109, 24071-24076.

[9] R. Car, M. Parrinello, Phys. Rev. Lett. 1985, 55, 2471.

[10] P. Silvestrelli, M. Parrinello, J. Chem. Phys. 1999, 111, 3572.

[11] P. L. Silvestrelli, F. Toigo, F. Ancilotto, J. Phys. Chem. B 2006.

[12] E. Fois, A. Gamba, G. Tabacchi, S. Quartieri, G. Vezzalini, J. Phys. Chem. B 2001, 105, 3012-3016.

[13] E. Fois, A. Gamba, A. Tilocca, J. Phys. Chem. B 2002, 106, 4806-4812.

[14] V. A. Ermoshin, K. S. Smirnov, D. Bougeard, Chem. Phys. 1996, 202, 53-61.

[15] P. Bornhauser, D. Bougeard, J. Phys. Chem. B 2001, 105, 36-41.

[16] R. Resta, Rev. Mod. Phys. 1994, 66, 899.

[17] N. Marzari, D. Vanderbilt, Phys. Rev. B 1997, 56, 12847.

[18] P. L. Silvestrelli, M. Bernasconi, M. Parrinello, Chem. Phys. Lett. 1997, 277(5-6), 478-482.

[19] R. Iftimie, M. E. Tuckerman, J. Chem. Phys. 2005, 122(21), 214508.

[20] R. Ramrez, T. Lopez-Ciudad, P. Kumar, D. Marx, J. Chem. Phys. 2004, 121, 3973-3983.

[21] M.-P. Gaigeot, M. Sprik, J. Phys. Chem. B 2003, 107, 10344.

[22] N. Desbiens, I. Demachy, A. H. Fuchs, H. Kirsch-Rodeschini, M. Soulard, J. Patarin, Angew. Chem. Int. Ed. 2005, 44, 5310-5313. 
[23] V. Eroshenko, R.-C. Regis, M. Soulard, J. Patarin, J. Am. Chem. Soc. 2001, 123, 8129-8130.

[24] P. L. Silvestrelli, M. Parrinello, Phys. Rev. Lett. 1999, 82, 3308-3311.

[25] K. Shirono, H. Daiguji, Chem. Phys. Lett. 2006, 417, 251-255.

[26] A. Luzar, D. Chandler, Nature 1996, 379, 55-57.

[27] I. A. Beta, H. Böhlig, B. Hunger, Phys. Chem. Chem. Phys. 2004, 6, 1975-1981.

[28] Y. Huang, Z. Jiang, Microporous Mater. 1997, 12, 341-345.

[29] P. B. Miranda, Y. R. Shen, J. Phys. Chem. B 1999, 103, 3292-3307.

[30] CPMD, Copyright IBM Corp 1990-2001, Copyright MPI für Festkörperforschung Stuttgart 19972005.

[31] A. D. Becke, Phys. Rev. A 1988, 38, 3098.

[32] C. Lee, W. Yang, R. G. Parr, Phys. Rev. B 1988, 37, 785.

[33] C. Baerlocher, W. M. Meier, D. H. Olson, Atlas of zeolite framework types; 2001.

[34] S. Nosé, J. Chem. Phys. 1984, 81, 511.

[35] W. G. Hoover, Phys. Rev. A 1985, 31, 1695.

[36] G. J. Martyna, M. E. Tuckerman, M. L. Klein, J. Chem. Phys. 1992, 97, 2635.

[37] M. Sharma, R. Resta, R. Car, Phys. Rev. Lett. 2005, 95, 187401.

[38] M. Gaigeot, R. Vuilleumier, M. Sprik, D. Borgis, J. Chem. Theory Comput. 2005, 1, 772. 